\title{
Brasil-Europa y la reestructuración del poder internacional*
}

I. EL SENTIDO DEL CONCEPTO DE REESTRUGTURACIÓN DEL PODER INTERNAGIONAL Y SU ADEGUACIÓN A LA REALIDAD CONTEMPORÁNEA

La principal dificultad que presenta la idea de la reestructuración del poder internacional como un modo de describir la realidad contemporánea es la de que ella sugiere, al mismo tiempo, el dinamismo y el estancamiento como las tendencias dominantes de la política internacional. De un lado, está el tema de la reestructuración, o sea, del cambio profundo, de la transformación de las estructuras internacionales; del otro, el énfasis en el poder, cuyas configuraciones se han revelado notablemente estables, tanto en el plano Este-Oeste cuanto en el Norte-Sur.

En esos dos ejes convencionales de la polftica internacional -el Este-Oeste y el Norte-Sur- se registran muy pocas transformaciones fundamentales en los últimos veinte años: el equilibrio global de poder estratégico continúa siendo hoy, como en la década del sesenta, la característica esencial del eje Este-Oeste en cuanto el desequilibrio, la asimetría de poder político y económico, continúa separando el Norte del Sur. A propósito, el Ministro Saraiva Guerreiro tuvo ocasión de declarar que "la evolución de la vida internacional está bloqueada por la consagración estructural de la desigualdad"1. El énfasis en la noción de poder modera fuertemente el contenido transformador de la idea de reestructuración internacional.

Si desmenuzáramos la naturaleza de los fenómenos de cambio

\footnotetext{
*El presente texto fue una contribución personal del autor al Seminario "BrasilEuropa Occidental: Cooperación y Conflicto", y no significa ninguna posición oficial en relación con los temas tratados.

${ }^{x}$ Ver conferencia pronunciada por el Embajador Ramiro Saraiva Guerreiro, Ministro de Estado de Relaciones Exteriores, en la Escuela Superior de Guerra el 5 de septiembre de 1980. Para una evaluación reciente de la actitud brasileña en cuanto al poder internacional y su impacto en la política externa, ver también la charla del Ministro Guerreiro "Líneas Básicas de Implementación de la Política Externa", pronunciada el 14 de agosto de 1981 con ocasión de la clausura del IV Seminario sobre Política Exterior del Brasil, organizado por la Facultad de Derecho de la Universidad de Minas Gerais.
} 
más flagrante en las relaciones internacionales -la emergencia de Europa Occidental y del Japón, por un lado, y del Tercer Mundo (en verdad, tan sólo de algunos de los países del Tercer Mundo, principalmente los exportadores de petróleo), del otro- vamos a verificar que, en ambos casos, no estuvo en juego la cuestión de poder en su forma más acabada. Es claro que me refiero al poder de destruir, de infligir daño, que se expresa en términos esencialmente militares y que, en último análisis, hoy es de naturaleza nuclear. En ese nivel, ni Europa Occidental, ni el Japón (en el contexto, la capacidad nuclear francesa es marginal), ni el Tercer Mundo, constituyen una amenaza al ordenamiento político-estratégico mundial, que continúa siendo determinado predominantemente por las superpotencias.

Seguramente, el poder es el más estático de los componentes de la ecuación internacional. Por eso mismo, los países que lo detenten están siempre sujetos a las tentaciones de la política de congelamiento del poder internacional, que el Embajador Araujo 'Gastro ${ }^{2}$ tuvo ocasión de analizar. Para esos países todo sucede como si la estabilidad de las relaciones de poder, como realidad última de la política internacional, generase no sólo derechos y deberes especiales (como efectivamente los genera en sentido limitado), sino que también autorizase la creencia de que el modo natural de conducción responsable de la polftica internacional es su centralización en las manos de las superpotencias.

En este último plano se puede hablar en forma realista sobre un proceso de reestructuración de la política internacional configurado por la emergencia, en un mundo desarrollado y uno en desarrollo, de núcleos de presencia política que escapan a los parámetros estrechos del poder, o sea, los de la confrontación o los del condominio global por parte de las superpotencias. La reducción de la política internacional a los términos de poder (y por lo tanto, a esa confrontación o a ese condominio) se revela como no necesaria, incómoda y finalmente contraproducente, tanto para Europa Occidental cuanto para el Tercer Mundo.

II. El MODELO POR EL GUAL SE PUEDE VER EN EL BRASIL EL PAPEL DE LOS PAÍses DE EUROPA OCGIDENTAL EN EL PROCESO DE REESTRUCTURACIÓN GLOBAL

Mi segundo comentario se refiere al modo por el cual podemos ver, en el Brasil, la participación de los europeos occidentales en la reestructuración internacional, tal como fue definida anteriormente.

Por lo común, cuando se aborda esa cuestión, se coloca el énfasis en las amplias posibilidades europeas de actuación internacional. Se

2Ver el volumen Araujo Castro, de la Colección Itinerarios, que acaba de ser publicado por la Editorial Universidad de Brasilia, especialmente página 197. 
hace una referencia, obligatoriamente, al hecho de que la expansión de esas posibilidades estaba ligada al crecimiento explosivo de la economía europea hasta hace algunos años, el cual, por depender del funcionamiento normal y estable de la economia internacional, ya sea en términos de insumos o bien de mercados, prácticamente obligó a Europa a elevar sus ojos para contemplar el estado del mundo y la obligó también a aumentar el grado de su compromiso político con los países en desarrollo

En esta oportunidad valdría la pena explicitar algunos límites a la capacidad de actuación europea en el proceso de transformación, en el cual, sin desearlo, Europa es uno de los actores claves:

1) En décadas pasadas, la primera limitación sería llamada geopolitica, pero hoy la rotulamos de estratégica. Las posibilidades europeas de movilizarse para actuar de forma autónoma en el plano mundial están severamente limitadas por el hecho de que, al mismo tiempo, Europa está presionada, incluso geográficamente, por una de las superpotencias $y$ se constituye en la principal frontera externa de la otra. Por sus implicaciones estratégicas y hasta ideológicas, ese simple hecho conturba la política externa de los países europeos, de modo que afecta drásticamente el perfil de su comportamiento externo;

2) El segundo límite se refiere a la propia identidad europea en el plano internacional. Por un lado, es ampliamente compartida la percepción de que cada país europeo puede, aisladamente, desempeñar un papel significativo en las principales cuestiones internacionales, pero muy raramente tendrá un peso decisivo; por otro lado, la actuación unificada de los diez de la comunidad europea, que suma influencias, dependen de un complicado y no siempre eficiente proceso de ajuste de posiciones e intereses. Además de eso, su propia naturaleza multilateral puede volver opaca, por así decir, la responsabilidad por las posiciones adoptadas;

3) La tercera es una limitación de credibitidad. Teniendo en vista la retórica recientemente empleada por los medios políticos $e$ intelectuales europeos, de la cual el Informe Brand ${ }^{\mathbf{3}}$ (rescatados sus aspectos positivos) es un ejemplo notable, se formó la impresión de que Europa podría, en el plano gubermamental, contribuir en forma realmente relevante a agilizar el diálogo Norte-Sur, con vistas a un reordenamiento de la economía internacional en términos más equitativos para los países en desarrollo. Esa impresión se está disipando rápidamente $\mathrm{y}$, en Iugar de ella, comienza a predominar la

sPara una evaluación del Informe Brandt ver, por ejemplo, Towards Due World?, publicado por la Fundación Friedrich Ebert en 1981; para una visión brasileña ver Roberto Abdenur y Ronaldo Sardenberg, Notas sobre las Relaciones Norte-Sur y el Informe Brandt, contribución a título personal en el Seminario Internacional sobre el Informe de la Comisión Brandt y sus Consecuencias para América Latina, Ganela, RGs, agosto de 1980 (publicado por la revista Estudios Internacionales, abril-junio 1981). 
percepción de que la supuesta flexibilidad europea está basada en la seguridad de que existe una inflexibilidad norteamericana, en el campo de la cooperación internacional, para el desarrollo y el reordenamiento de las estructuras económicas internacionales. En otras palabras, Europa se puede permitir gestos de conciliación con relación al Sur, porque está cierta de que las conversaciones NorteSur no prosperarán debido a la inflexibilidad norteamericana.

4) Una cuarta y última limitación sería de sensibilidad. Descontada nuevamente la retórica; la sensibilidad de los países centrales de Europa en relación a la periferia del Tercer Mundo no se va relevando tan pura como podría esperarse si los países europeos estuviesen realmente interesados en desempeñar un papel diferenciado dentro de Occidente. La visión europea está excesivamente restringida a sus intereses económicos más inmediatos, en un cuadro marcado por políticas proteccionistas a nivel nacional y comunitario. Los países europeos todavía son reacios a establecer un amplio diálogo político con los países del Sur. Persisten, en lo esencial, en exagerar en el mundo en desarrollo, no un semejantie sino un otro, hostil. y exótico. Tal vez la razón fundamental de esa actitud radica en la propia relación histórica entre Europa y el Tercer Mundo. Tal vez el colonialismo haya dejado en el alma europea marcas más profundas de las que se osa imaginar, marcas culturales todavía no cicatrizadas enteramente.

El resultado global de esas limitaciones es que en cuanto los Estados Unidos y la URss se presenten o procuren presentarse en la escena internacional como polos de atracción excluyentes que obligarían a los diferentes estados a opciones políticas, Europa no tendría condiciones ni siquiera potenciales para desempeñar un papel semejante, aunque actuara en forma unificada. Tampoco dentro de Occidente puede Europa constituir una alternativa a los Estados Unidos; ella representa sólo un interlocutor más un nivel complementario de conexión política y económica. En ese sentido, la contribución europea a la transformación de las estructuras mundiales tenderá, desgraciadamente, a ser mucho menor que lo que su experiencia política y su poderío económico autorizarían a pensar.

En el sentido más amplio, la participación activa de Europa en el proceso de reordenamiento internacional es más necesaria que nunca. Uno de los elementos más urgentes y cruciales de ese proceso se sitúa en el eje Norte-Sur y supone un amplio y coordinado esfuerzo político de parte de Occidente para recuperar la confianza del Tercer Mundo. Dadas las realidades políticas contemporáneas, si Europa Occidental trascendiese sus limitaciones y vulnerabilidades, podría promover la reforma de las actitudes occidentales en relación a los tres continentes de América Latina, Africa y Asia.

Si bien la cooperación Norte-Sur estaba estancada, y recientes acontecimientos estaban contribuyendo al entorpecimiento del diálogo entre América Latina y Europa, pienso que no existe una opo- 
sición irremovible de intereses entre el Norte y el Sur. Pero es necesario que el Norte se disponga a dar un tratamiento más constructivo y abierto a las fajas de complementariedad o convergencia de ese diálogo para que se puedan alcanzar nuevos niveles de entendimiento.

Es, pues, imprescindible que Europa, Io mismo que los gobiernos y la opinión pública, muestren una actitud más nítida en favor de que el Occidente se redefina de modo que incluya en el ámbito de su convivencia $y$, consecuentemente, en sus procesos decisorios, no sólo las democracias industrializadas sino también los países del Sur que forman parte del mundo occidental, en razón de sus tradiciones históricas, concepciones filosóficas, aspiraciones políticas y vinculaciones económicas. (Se debe también abrir un espacio para impulsar el diálogo con los países del Tercer Mundo que, al menos económicamente, están ligados al Occidente y que, si bien no pueden ser considerados propiamente occidentales por sus tradiciones, opciones políticas o formas de organización social, deben contar con la posibilidad de una mayor aproximación con el Occidente, como también de un acceso más fácil y expedito al comercio, al desarrollo y al bienestar).

\section{III}

Mi tercer comentario se relaciona con el papel del Brasil en el proceso de reordenamiento internacional. Antes deseo señalar que los medios brasileños de acción y de influencia externa, ya sea en el campo político o bien en el económico, están claramente limitados por el hecho de que estamos orientados esencialmente hacia nuestro propio desarrollo. En el Brasil no hay medios fácilmente movilizables para la acción externa y por eso debemos ser cautelosos en las operaciones que emprendemos. No tenemos "sobras" militares o económicas para aminorar, compensar o corregir errores políticos. El capital político de que disponemos, especialmente en el Tercer Mundo, fue acumulado exclusivamente a costa nuestra y por nuestros propios esfuerzos. La prudencia manda, pues, que no nos disipemos y que sólo nos comprometamos cuando es posible vincular claramente nuestras acciones diplomáticas con nuestros intereses específicos.

Una vez hechas esas precisiones, diría que la participación del Brasil en el proceso de reestructuración internacional está condicionada por la posición del país frente a Occidente y al Tercer Mundo. Inclusive la más superficial lectura de la realidad brasileña revelaría que, junto a fuertes trazos occidentales, otros con raíz en el Tercer Mundo marcan nuestra vida nacional.

Sin embargo, hay quienes ocasionalmente intentan negar este último componente de la herencia brasileña, como si el mismo fuera materia de opción y no una consecuencia de nuestras realidades 
socioeconómicas, de nuestra inserción latinoamericana y de la presencia africana en nuestra cultura y sociedad. Lo más curioso es que en el exterior, entre los países occidentales industrializados, la percepción francamente dominante es la de que el Brasil y América Latina son parte del Tercer Mundo. Todo sucede como si aquellos paises tuvieran dificultad para reconocer el carácter occidental de las sociedades latinoamericanas.

El profesor Helio Jaguaribe hace avanzar significativamente esa cuestión, al observar en un estudio reciente ${ }^{4}$, que la condición occidental para el Brasil proviene de su. cultura implantada por los descubridores y desarrollada en estrecho contacto con Europa y no de sus posiciones políticas..." y añade con objetividad que "no es por concesiones de terceros que el Brasil es un país occidental. Tal condición no es otorgable por ninguna negociación". Jaguaribe resalta además que "pertenecer al Occidente no confiere per se la condición de miembro del Primer Mundo".

Utilizando esa matriz conceptual, haría tres observaciones relacionadas con nuestro tema central:

i) El Brasil es parte de Occidente y desea ser miembro del Primer Mundo. La actuación brasileña en relación a la transformación de las reglas de juego internacionales tendrá que tomar en cuenta ese desideratum nacional de largo plazo. Se entiende el Primer Mundo como un mundo de libertad política individual y bienestar socioeconómico para todos los estratos de población (ésta es, tal vez, una visión idealizada), pero esto está fuera de nuestro universo de discrasión en este momento;

ii) El Brasil es un país occidental, pero posee un patrimonio cultural fuerte y propio, en el cual éstán mezcladas influencias occidentales y tercermundistas. Esa variada herencia cultural nos distingue de los países occidentales que hoy en día están industrializados. Pienso que la dimensión del Tercer Mundo continuará estando presente en la sociedad brasileña incluso cuando llegue el momento, tal vez utópico, en que un nuevo Brasil plenamente industrializado y democrático se reconozca, y sea reconocido, como miembro del Primer Mindo. En esas condiciones me parece estéril un debate sobre si el Brasil dejará un día de ser un país del Tercer Mundo, debate que implica la ilusión de que el pais sólo está en el Tercer Mundo, pero no es del Tercer Mundo. La manera de preservar nuestra autenticidad es tener conciencia de nuestras condiciones peculiares e incorporarlas como componentes de nuestra identidad. Si me fuese permitido hacer un paralelo, diría que así como la sociedad brasileña no se "blanquea", el Brasil no se "occidentaliza";

Helio Jaguaribe: O Futuro das Relacoes Brasil-Estados Unidos, mimeografiada, 1981. 
iii) La complejidad de nuestro patrimonio histórico y cultural nos proporciona una aguzada sensibilidad para lo que debiera ser la actitud de Occidente en relación al Tercer Mundo, en el proceso de reestructuración internacional. El Brasil debe formas de solidaridad al Tercer Mundo porque eso corresponde, primero, a un dato cultural nuestro como país latinoamericano y de influencia africana $\dot{y}$, segundo, al interés económico brasileño, teniendo en cuenta el creciente desarrollo comercial del país con el mundo en desarrollo. Si el Tercer Mundo va mal económicamente, mayores serán las dificultades del Brasil para obtener sus metas de exportación y de desarrollo.

A partir de esas posiciones, tenemos interés en que sea revitalizado el concepto de Occidente, de modo de incorporar no sólo la visión de los beneficios del status quo internacional sino también el enfoque crítico y revisionista, más constructivo, de países como el Brasil. Justamente por no estar entre los países desarrollados, si bien somos parte de Occidente, es que tenemos buenas condiciones para interpretar los rumbos y los verdaderos intereses de la gran comunidad occidental en esta fase de transformación del orden internacional.

\section{IV}

Añadiré algunos comentarios sobre la naturaleza y las perspectivas de las relaciones Biasil-Europa Occidental en el contexto de la reestructuración internacional. No necesito examinar los aspectos económicos de esas relaciones, toda vez que ya fueron tratados en otras oportunidades y existe amplia literatura sobre los mismos.

Hay un cambio en la visión que tiene Europa Occidental en sus relaciones con el Brasil y con América Latina, de modo general. Por ejemplo, Stanley Hilton ${ }^{B}$ expresa que, desde fines del siglo xIx se notaba una falta de interés político de Europa Occidental por América Latina, salvo episodios como el de Napoleón III en México, que acabó mal. Hilton observa que la política del Reino Unido para América Latina, a fines del siglo pasado, ya era vista como mera resultante de la política británica en relación a los Estados Unidos y de éstos en cuanto al Reino Unido. De alguna forma, esta observación corrobora la opinión de Celso Lafer7: el orden regional era determinado a partir de las relaciones entre las dos grandes potencias de la época, y el papel de por lo menos una

Ver, por ejemplo, Walder de Gós, Brazil Turus to Western Europe: Changing Perspectives, mimeografiada, 1981.

Stanley Hilton, Latin America and Western Europe, 1881-1945: The Political Dimension, mimeografiada, 1981.

TEn intervención en el Foro San Thiago Dantes, en junio de 1981. 
de ellas, la menos interesada en América Latina, era determinado a partir del orden mundial, organizado, por así decir, en forma de condominio de los Estados Unidos y del Reino Unidos.

En nuestro siglo hubo una creciente concentración de la atención europea en los aspectos económicos de las relaciones con América Latina, con excepción, en parte, de la política de Alemania antes de la Segunda Guerra Mundial. Como consecuencia de ese enfoque, sè registraron grandes ganancias por parte de los países europeos, en el sentido de que se libraron de incómodas y costosas obligaciones político-militares. Por otro lado, esa situación reflejaba el gradual debilitamiento político-militar de Europa, al paso que afirmaba la autoridad política de los Estados Unidos en Occidente.

G. D. Kornat $t^{9}$ notó que en la década de los 50, Europa Occidental vivía a una "distancia astronómica" de América Latina; los lazos comerciales se habrían restablecido, pero fuera de eso, había principalmente preocupaciones científicas y de cuna antropológica y etnológica. Las sociedades "primitivas" de América Latina eran vistas como "contraparadigmas" de las sociedades avanzadas de Europa Occidental.

A fines de la década del 50, esa situación comenzó a cambiar rápidamente y Kornat correlaciona ese cambio con el proceso de descolonización, con lo que tiende a concordar. Desde el momento en que fueron puestos en discusión los lazos entre Europa y el mundo colonial, especialmente el Africa, era natural que fuesen buscados nuevos contactos.

En este nuevo abordaje en cuanto a América Latina, Europa Occidental pasó de la curiosidad científica a la teoría del desarrollo. Nótese, pues, que en esa transición los países latinoamericanos no eran vistos como socios políticos, ni siquiera potenciales, sino sólo como áreas que debían ser "desarrolladas". No se les aceptaba como entidades con las cuales Europa Occidental pudiese tener una relación política real o efectiva.

En esa época se inicia una forma de transferencia de ideología o, más bien, de transferencia de tecnología político-partidaria. En América Latina se organizó el movimiento demócrata-cristiano el cual, durante mucho tiempo, fue símbolo de esa transferencia de una visión política europea para nuestra región. Hoy, ese símbolo es el desarrollo de la Internacional Socialista de América Latina, en otro plano y de forma diferente.

La crisis mundial iniciada en 1973 va a introducir en ese panorama elementos radicalmente nuevos. A partir de aquel año, la vida internacional se torna más complicada, no sólo para América Lati-

'Los alemanes, desde el inicio de este siglo, tendfan a encarar la actitud norteamericana en relación a América Latina como exclusivista. Giertamente los alemanes no se beneficiaban tanto del orden mundial y regional como los ingleses.

${ }^{\circ} \mathrm{G}$. D. Kornat, Latina America Rediscavered, mimeografiada, 1981. 
na (y el Brasil), sino también para los países europeos. En aquella lucha por la "apropiación del producto mundial"10, exacerbada por la dramática alza de los precios del petróleo, los europeos comienzan a percibir, aunque precariamente, que había fallado ese modelo de relacionarse con América Latina y el Tercer Mundo, de modo general, sólo como área de inversión y de consumo de productos exportados por los países desarrollados y como área abastecedora de recursos naturales.

Gradualmente, Europa Occidental, con Alemania al frente, politiza sus relaciones con el Tercer Mundo, inclusive el Brasil. Ese proceso es visible, en términos políticos, por ejemplo, en Africa, y. hoy es mucho mayor que en 1973. Basta ver el desarrollo de los países sin tradición colonial reciente en las cuestiones de Zimbabue y de Namibia.

El grado de interés político europeo por el Brasil comenzó a cambiar, inclusive porque somos unos de los países en desarrollo que tienen posibilidades de participar, en alguna forma, del reordenamiento internacional, inaugurado con la crisis de 1973. Esta politización es también parte del esfuerzo de legitimación de la acción económica: para los europeos occidentales se torna mucho más legitimo postular, junto con los países del Sur, relaciones económicas aumentadas a partir de una báse política más amplia. Existe también preocupación por llenar los espacios vacíos dejados por los Éstados Unidos.

Por el lado del Brasil se registra una diversificación de nuestras relaciones exteriores: no sólo iniciamos la reforma de nuestros relaciones con Europa Occidental, con la búsqueda directa de entendimientos políticos a partir de las visitas presidenciales: en 1967 a Inglaterra y a Francia y después, en 1978, Alemania, las que obtuvieron grados variados de respuesta 11 , sino que también incorporamos los horizontes africanos y árabes a nuestra política internacional. Superamos la etapa declaratoria y pasamos a las relaciones directas e inmediatas con árabes y africanos. En la actualidad ese proceso prosigue sin desfallecimiento.

Todo esto estará unido al cambio y diversificación del sistema económico del Brasil. En el Tercer Mundo, el Brasil es el paîs de grandes dimensiones con la estructura económica más diversificada, con la pauta de exportaciones más variada y el que tiene el mayor

\footnotetext{
${ }^{10} \mathrm{La}$ expresión es del Canciller Helmut Schmidt, en un artículo de la revista Foreign Affairs, de abril de 1974.

Incluso antes de los viajes presidenciales, ya había perfecta conciencia de la necesidad de reestructurar las relaciones entre Brasil y Europa Occidental. Ver, por ejemplo, el discurso pronunciado por el entonces Canciller Azeredo da Silveira con ocasión de la visita al Brasil del Ministro de Asuntos Extranjeros de Francia, en enero de 1976 o las palabras, también del Embajador Azeredo da Silveira, ante las Comisiones de Relaciones Exteriores del Senado Federal y de la Cámara de Diputados en agosto del mismo año.
} 
número de clientes. Esa circunstancia nos lleva a abandonar los senderos estrechos de las relaciones basadas en la exportación de pocos productos de base para pocos mercados, en favor de unas relaciones externas más universales.

En ese contexto, el hecho fundamental es el de la complejidad y, recogiendo lo que dice Celso Lafer ${ }^{12}$, nuestra preocupación debe ser la de evitar el empobrecimiento conceptual. Tenemos que huir de las simplificaciones atrayentes, por ejemplo, el modelo de diversificación de la dependencia, que es simple. aunque estático. Y con un claro sesgo pesimista. Sería igualmente insuficiente explicar la activación y la diversificación del verfil externo del Brasil exclusiva o preponderantemente a partir de factores "exógenos" al pafs, o adoptar la tesis mecanicista de que las relaciones del Brasil oscilarfan como un péndulo entre el Primero y el Tercer Mundo, etc. No serfa suficiente ni adecuado intentar explicar lo que se está haciendo en materia de política externa en términos de los constreñimientos estructurales de la realidad internacional, restricciones que se está tratando de superar o, por lo menos, de compensar, por variados caminos.

A pesar de los progresos alcanzados, la conexión entre el Brasil y Europa Occidental todavfa no ha realizado la mayor parte de sus potencialidades. En el nivel más amplio de la sociedad no se nota en los medios europeos, salvo raras excepciones, una mayor receptividad en el plano de Ias ideas, al Brasil y a América Latina en general, y el intercambio intelectual permanece excesivamente desequilibrado en favor de Europa. En el plano oficial se puede registrar como factor nuevo y positivo en lo que se refiere al Brasil, la realización de consultas polfticas con algunos factores europeos.

Tanto en uno como en otro nivel hay deficiencias obvias en relación a la frecuencia y a la profundidad del examen conjunto de temas, como los de hoy, referentes al reordenamiento de la vida internacional. Son los temas que comprenden las reglas de convivencia entre los países $y$, en último análísis, la paz y la seguridad internacionales. En ese contexto, tal vez valiese la pena examinar en la fase de debates la hipótesis de que en Europa existirian resistencias no declaradas al proceso de transformación global y no habría un interés suficientemente fuerte en abordar esos temas, de forma operativa, con los países de América Latina.

En ló que se refiere a las reläciones entre el Brasil y Europa, en el contexto de la transformación de las estructuras internacionales diría, a título de comentario final, que ambas partes tendrían mucho que ganar si se afirmase la percepción de que la política externa brasileña guarda su especificidad, o sea, está directamente

Ian la misma ocasión mencionada en la nota 7. 
Francisco Orrego Dicuña / La definición de un régimen para los recursos...

conectada con los intereses concretos del Estado el que, en esas condiciones, no es instrumentable. La política externa responde a los intereses brasileños y son ellos los que primordialmente determinan su ritmo, contenido y dirección. 